\title{
ÉPREUVE Au BLEU de MÉTHYLène ET QUALITÉ BACTÉRIOLOGIQUE DU LAIT (1)
}

\author{
par \\ Roger POFFÉ, Maurice WECKX et Paul SIMONART \\ Station de Recherches Laitières \\ Université de Louvain, Heverlee-Louvain
}

\section{Introduction}

En vue de promouvoir la qualité de la matière première livrée aux laiteries, un arrêté royal (14-11-1963) réglemente en Belgique, le paiement du lait selon sa qualité. Sur base de l'épreuve au bleu de méthylène, il répartit les laits en trois catégories, dont la première jouit d'une prime tandis que la troisième subit une réfaction. L'indice de qualité varie avec la température extérieure et, dès lors, le système ne rétribue pas, ou ne pénalise pas, la qualité intrinsèque du lait, mais bien sa qualité relative.

La présente étude porte sur la qualité bactériologique de 1000 échantillons de lait, déterminée comparativement par l'épreuve au bleu de méthylène et par le dénombrement microscopique des cellules bactériennes. Elle confronte les résultats ainsi obtenus avec ceux que donne le système de classification des laits instauré par la réglementation belge. Celle-ci couplant le résultat de l'épreuve au bleu de méthylène à la température extérieure, il paraissait utile d'établir l'influence de cette température sur la qualité bactériologique intrinsèque du lait.

\section{Méthode expérimentale}

Les 1000 échantillons de lait étudiés ont été prélevés en laiterie du 17 septembre 1964 au 20 janvier 1965, dans le bac de la bascule réceptrice. Il s'agit donc d'échantillons moyens d'un producteur, ou d'échantillons de "petit mélange ".

Les échantillons, prélevés en flacons stériles, étaient immédiatement acheminés au laboratoire et aussitôt examinés.

(1) Travail effectué sous les auspices de l'I.R.S.I.A. (Institut de la Recherche Scientifique pour l'Industrie et l'Agriculture) selon le programme du Comité pour l'étude scientifique et technique du lait. 
L'épreuve au bleu de méthylène s'effectue à $37^{\circ} \mathrm{C}$ avec inversion. des tubes toutes les heures. Quant à la méthode de Breed, elle a été appliquée selon les prescriptions de "Standard methods for the examination of dairy products $)[10]$.

Le lait “ $A$ » est celui qui, outre qu'il doit satisfaire à d'autres prescriptions, est refroidi immédiatement après la traite à une température inférieure à $15^{\circ} \mathrm{C}$.

La classification des laits en trois catégories en fonction de la température extérieure à $10 \mathrm{~h}$ du matin et du résultat de l'épreuve au bleu de méthylène, tel que la prévoit la réglementation en question, est la suivante :

\begin{tabular}{|c|c|c|c|}
\hline \multirow[t]{2}{*}{$\begin{array}{l}\text { Température } \\
\text { extérieure }\end{array}$} & \multicolumn{3}{|c|}{$\begin{array}{c}\text { Temps nécessaire } \\
\text { à obtenir la décoloration du bleu de méthylène }\end{array}$} \\
\hline & $\begin{array}{l}\text { Première } \\
\text { catégorie }\end{array}$ & $\begin{array}{l}\text { Deuxième } \\
\text { catégorie }\end{array}$ & $\begin{array}{l}\text { Troisième } \\
\text { catégorie }\end{array}$ \\
\hline 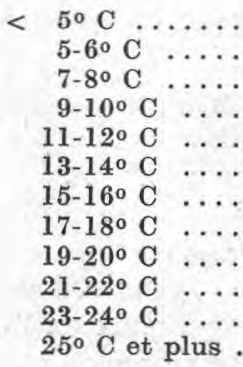 & $\begin{array}{l}5 \text { h } 00 \text { ou plus } \\
4 \text { h } 30 \text { ou plus } \\
4 \text { h } 00 \text { ou plus } \\
3 \text { h } 35 \text { ou plus } \\
3 \text { h } 15 \text { ou plus } \\
2 \text { h } 45 \text { ou plus } \\
2 \text { h } 20 \text { ou plus } \\
2 \text { h } 00 \text { ou plus } \\
1 \text { h } 35 \text { ou plus } \\
1 \text { h } 15 \text { ou plus } \\
1 \text { h } 05 \text { ou plus } \\
1 \text { h } 00 \text { ou plus }\end{array}$ & 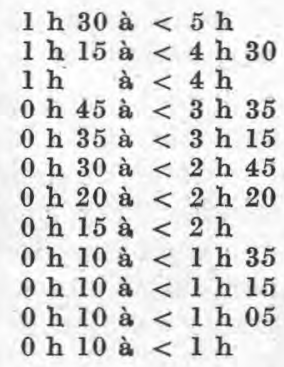 & $\begin{array}{l}<1 \mathrm{~h} 30 \\
<1 \mathrm{~h} 15 \\
<1 \mathrm{~h} \\
<0 \mathrm{~h} 45 \\
<0 \mathrm{~h} 35 \\
<0 \mathrm{~h} 30 \\
<0 \mathrm{~h} 20 \\
<0 \mathrm{~h} 15 \\
<0 \mathrm{~h} 10 \\
<0 \mathrm{~h} 10 \\
<0 \mathrm{~h} 10 \\
<0 \mathrm{~h} 10\end{array}$ \\
\hline
\end{tabular}

\section{Résultats et discussion}

En ce qui concerne la corrélation entre le temps de décoloration du bleu de méthylène et le clump count, le tableau I donne les moyennes statistiques, les déviations standard, les coefficients de corrélation et les lignes de régression. La figure 1 représente graphiquement ces données.

Selon $y=f(x)$, c'est-à-dire l'influence de l'épreuve du bleu de méthylène sur le clump count, les observations suivantes se dégagent. La ligne de régression de la totalité des échantillons, 
ultérieurement désignée par "ligne de régression totale ", indique que pour un temps de réduction moyen de $3,9 \mathrm{~h}$, on peut escompter un clump count de 1200000 . Pour un temps de réduction de $8 \mathrm{~h}$, on escompte un clump count de 77000 , mais le " confidence interval " de 5 p. 100 varie de 12000 à 500000 . Pour un temps de réduction de $5 \mathrm{~h}$, le clump count sera de 550000 avec une dispersion allant de 86000 à 3500000 . Les valeurs correspondantes pour un temps de réduction de $3 \mathrm{~h}$ seront : 2000000,320000 et 13000000 . Enfin, pour un temps de réduction de 1 heure, ces valeurs seront respectivement 7600000,1200000 et 48000000 .

La ligne de régression des laits de première catégorie s'écarte de la ligne de régression totale et la coupe à $5,75 \mathrm{~h}$ et au clump count de 300000 . Il s'en suit que pour un temps de réduction de l'ordre de $6 \mathrm{~h}$, on doit escompter un clump count plus élevé que celui qui correspond à la ligne de régression établie pour la totalité des échantillons.

La ligne de régression des laits de seconde catégorie est à peu près parallèle à celle de la ligne de régression totale, mais elle se situe en dessous de celle-ci. Il en résulte qu'ici, à l'inverse de ce qui vient d'être dit pour les laits de première catégorie, c'est un clump count moins élevé qui correspondra à un temps de réduction donné.

La ligne de régression des laits de troisième catégorie s'écarte sensiblement de la ligne de régression totale. Pour un temps de réduction donné, le clump count correspondant sera plus élevé pour ces laits que pour la totalité des échantillons. La réduction instantanée du bleu de méthylène correspond à un clump count de 55000000 .

Si on considère $x=f(y)$, ou l'influence du clump count sur le temps de réduction, on observe ce qui suit :

D'après la ligne de régression totale un elump count de 1200000 fait escompter un temps de réduction de $3,9 \mathrm{~h}$. Un clump count de 100000 fait prévoir un temps de réduction de $6,4 \mathrm{~h}$ avec des écarts de 4,1 à 8,8 h. Pour un clump count de 1000000 , on peut escompter un temps de réduction de $4 \mathrm{~h}$, mais le "confidence interval " de 5 p. 100 varie de 1,7 à 6,4 h. Enfin, un clump count de 10000000 fait prévoir un temps de réduction de $1,6 \mathrm{~h}$ avec des limites de 0 à $4 \mathrm{~h}$.

Si, pour chacune des trois valeurs de clump count qui viennent d'être envisagées, on considère séparément les diverses catégories de lait, on arrive aux conclusions suivantes. Pour un clump count de 100000 le temps de réduction moyen est de $4,5 \mathrm{~h}$ lorsqu'il s'agit de lait de deuxième catégorie et de $6,3 \mathrm{~h}$ pour le lait de première catégorie.

Un clump count de 1000000 correspond à un temps de réduction de $3 \mathrm{~h}$ pour le lait de deuxième catégorie, et de $4,9 \mathrm{~h}$ pour celui de première catégorie. 
TABLEAU I

CORRÉLATION ENTRE LE TEMPS DE RÉDUCTION DU BLEU DE MÉTHYLÈNE ET LE CLUMP COUNT

\begin{tabular}{|c|c|c|c|c|c|c|c|c|c|}
\hline \multirow[b]{2}{*}{ Echantillon } & \multirow{2}{*}{$\begin{array}{l}\text { Nombre } \\
\text { d'échan- } \\
\text { tillons }\end{array}$} & \multicolumn{2}{|c|}{ Moyennes } & \multicolumn{2}{|c|}{$\begin{array}{l}\text { Déviation } \\
\text { Standard }\end{array}$} & \multirow{2}{*}{$\begin{array}{c}\text { Coefficient } \\
\text { de } \\
\text { corrélation }\end{array}$} & \multirow{2}{*}{\multicolumn{2}{|c|}{ Lignes de régression }} & \\
\hline & & $\begin{array}{c}\text { Temps de } \\
\text { réduction } \\
\text { (heures) } \\
x\end{array}$ & $\begin{array}{c}\text { Clump } \\
\text { Count } \\
(\log ) \\
y\end{array}$ & $S x$ & Sy & & & & \\
\hline Total ......... & 1000 & 3,9 & 6,06 & 2,08 & 0,72 & $-0,83$ & $y=7,16$ & $6-0,28 x x=18,45$ & $-2,41 y$ \\
\hline 1re catégorie ... & 520 & 5,5 & 5,58 & 1,29 & 0,45 & $-0,50$ & $y=6,52$ & $2-0,17 x x=13,46$ & $-1,43 y$ \\
\hline Lait ordinaire. & 443 & 5,6 & 5,58 & 1,27 & 0,46 & $-0,50$ & $y=6,59$ & $9-0,18 x x=13,32$ & $-1,38 y$ \\
\hline Lait A ..... & 77 & 4,7 & 5,58 & 1,10 & 0,39 & $-0,63$ & $y=6,62$ & $2-0,22 x x=14,78$ & $-1,81 y$ \\
\hline $2^{e}$ catégorie $\ldots$. & 398 & 2,4 & 6,42 & 1,00 & 0,45 & $-0,66$ & $y=7,14$ & $4-0,30 x x=11,85$ & $-1,47 y$ \\
\hline Lait ordinaire. & 352 & 2,5 & 6,43 & 1,03 & 0,47 & $-0,68$ & $y=7,19$ & $9-0,31 x x=12,15$ & $-1,51 y$ \\
\hline Lait $A \ldots \ldots$ & 46 & 2,0 & 6,38 & 0,62 & 0,32 & $-0,59$ & $y=6,97$ & $7-0,30 x x=9,25$ & $-1,14 y$ \\
\hline $3^{\mathbf{e}}$ catégorie $\ldots$. & 82 & 0,6 & 7,36 & 0,33 & 0,44 & $-0,44$ & $y=7,74$ & $4-0,59 x x=3,10$ & $-0,33 y$ \\
\hline
\end{tabular}




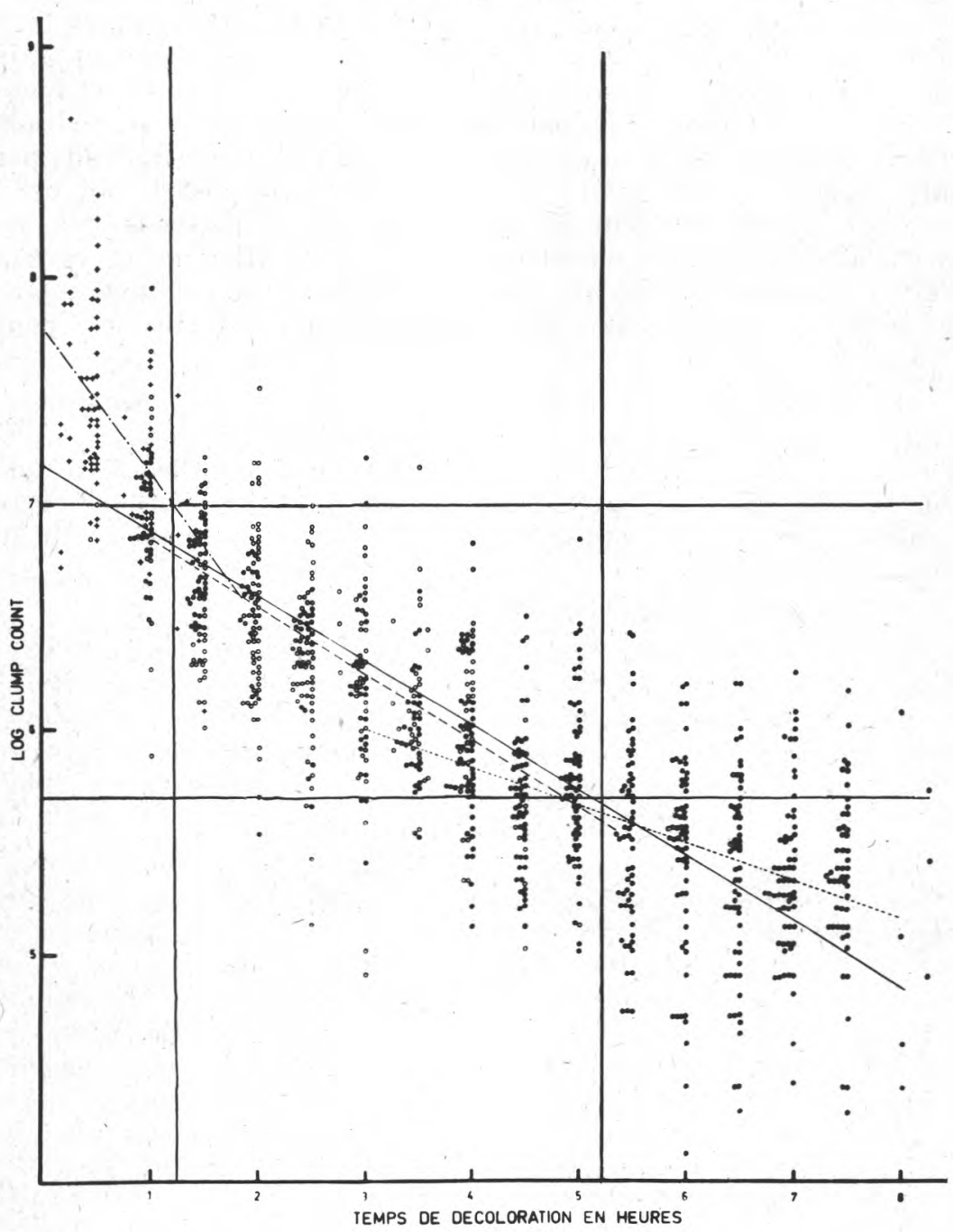

Fig. 1. - Corrélation entre le temps de décoloration du bleu de méthylène et le log. du Clump Count

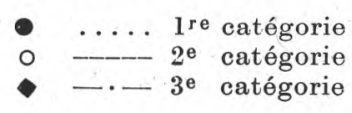

A un clump count de 10000000 correspond un temps de réduction moyen de $45 \mathrm{mn}$, de $1,6 \mathrm{~h}$ et de $3,5 \mathrm{~h}$, selon que l'on a à faire respectivement à des laits de troisième, de seconde ou de première catégorie. 
Le tableau I fait aussi la distinction entre laits " $A$ » et laits ordinaires. Ces deux types de lait, lorsqu'ils appartiennent à la première catégorie, ont une moyenne de clump count qui est identique, mais le temps de réduction moyen est différent. Celui-ci est de 5,6 pour le lait ordinaire et de 4,7 pour le lait A, dont, par ailleurs, les extrêmes sont plus rapprochées que pour le lait ordinaire. La différence d'inclinaison des lignes de régression de ces deux laits n'est pas significative tandis que la différence de niveau l'est. Ceci revient à dire que pour un même clump count, le temps de réduction moyen sera plus long pour le lait ordinaire que pour le lait A.

Pour l'ensemble des résultats, le coefficient de corrélation entre clump count et temps de réduction est égal à $-0,83$, ce qui correspond à la valeur de $-0,75$ trouvé par Dabbah et Olson [4]. Cela correspond aussi de façon satisfaisante aux corrélations entre " plate-count" et temps de réduction de $-0,85,-0,63$ et $-0,70$ citées respectivement par Overby [7], Thomé et al. [12] et par Nilsson et von Bockelmann [6].

La figure 2 représente graphiquement les pourcentages d'échantillons répartis, d'une part, en trois groupes selon le clump count et, d'autre part, en trois groupes selon l'épreuve au bleu de méthylène. Dans chacun de ces groupes, la distinction est, en outre, faite entre lait de première, de seconde et de troisième catégorie, selon la réglementation en vigueur.

Sur base du clump count, on observe que 32,9 p. 100 des échantillons appartiennent au groupe qui renferme moins de 500000 bactéries par $\mathrm{ml}$; 30,6 p. 100 sont des laits de première catégorie et 2,3 p. 100 sont des laits de seconde catégorie. Le second groupe qui renferme entre 500000 et 10000000 clumps par ml, représente 57,3 p. 100 des échantillons dont 21,3 p. 100 appartiennent à la première catégorie, 34,2 p. 100 à la seconde et 1,8 p. 100 à la troisième. Ceci revient à dire que 21,3 p. 100 des échantillons jouissent d'une prime de qualité alors qu'ils sont de qualité bactériologique médiocre. Le troisième groupe, soit 9,8 p. 100 d'échantillons avec plus de 10000000 clumps par ml, renferme 0,1 p. 100 d'échantillons de première catégorie, 3,4 p. 100 de deuxième et 6,3 p. 100 de troisième.

La répartition des échantillons selon le temps de décoloration montre ce qui suit. Dans le premier groupe (temps de décoloration supérieur à $5 \mathrm{~h}$ ) on trouve 30,3 p. 100 des échantillons qui tous, sont de première catégorie. Le second groupe (temps de décoloration entre 1 et 5 h) renferme 57,2 p. 100 des échantillons dont 21,7 p. 100 de première eatégorie, 35,0 p. 100 de deuxième et 0,5 p. 100 de troisième. Enfin, le troisième groupe, soit 12,5 p. 100 d'échantillons avec un temps de décoloration inférieur à $1 \mathrm{~h}$, ne contient que des échantillons de deuxième et de troisième catégorie. 
Les deux systèmes de répartition des échantillons que représente la figure 2, paraissent, à première vue, équivalents. En effet, le pourcentage total des échantillons dans chacun des trois groupes, est voisin pour les deux systèmes. Cependant, ces pourcentages ne portent pas sur les mêmes échantillons de lait. La répartition des échantillons dans chaque groupe selon la catégorie, varie d'après que l'on envisage le clump count ou le résultat de l'épreuve au bleu de méthylène. Cela ressort nettement de la figure 1, qui partage les laits en trois groupes selon deux lignes horizontales sur base du clump count, et selon deux lignes verticales sur base de l'épreuve au bleu de méthylène.

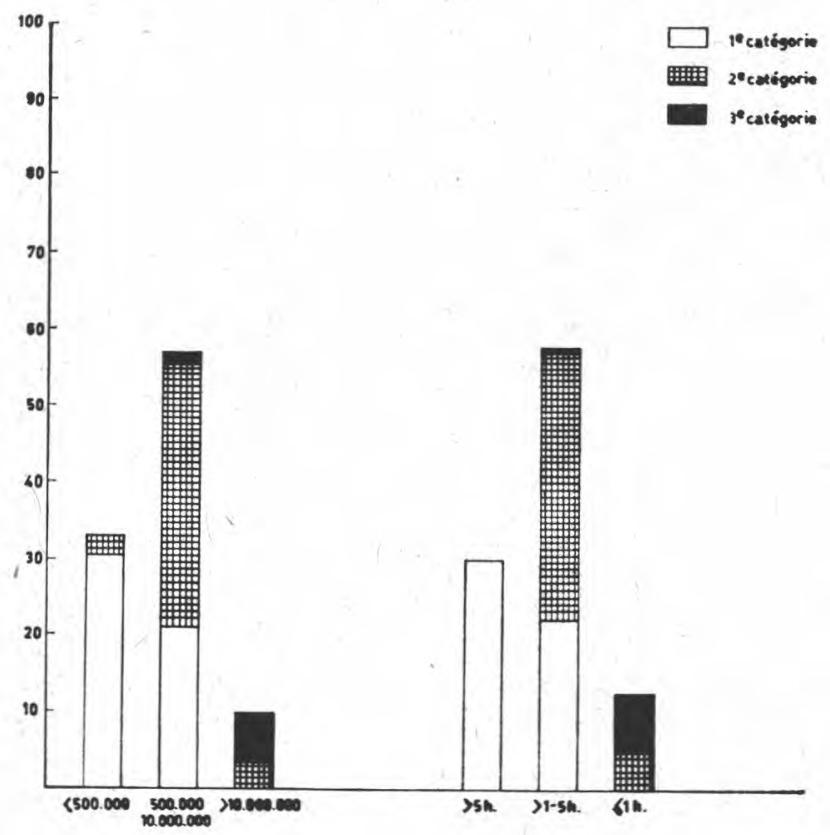

Fig. 2. - Pourcentages d'échantillons classés en trois catégories et répartis en trois groupes selon le Clump Count et en trois groupes selon l'épreuve au bleu de méthylène

S'il n'y a pas concordance entre temps de décoloration du bleu de méthylène et classification selon le système réglementaire en vigueur, c'est parce que celui-ci applique un facteur correctif en fonction de la température ambiante. C'est pourquoi il est utile d'examiner de plus près l'influence de cette température sur les résultats du clump count et sur ceux de l'épreuve au bleu de méthylène. 
En ce qui regarde l'influence de la température on constate, d'après la figure 3 , que le pourcentage de laits classés en première catégorie ne varie presque pas en fonction de la température extérieure et reste voisin de 50 p. 100 . Le nombre d'échantillons classés en deuxième catégorie varie entre 38,2 et 41,9 p. 100 et celui d'échantillons classés en troisième catégorie entre 7,3 et 11 p. 100.

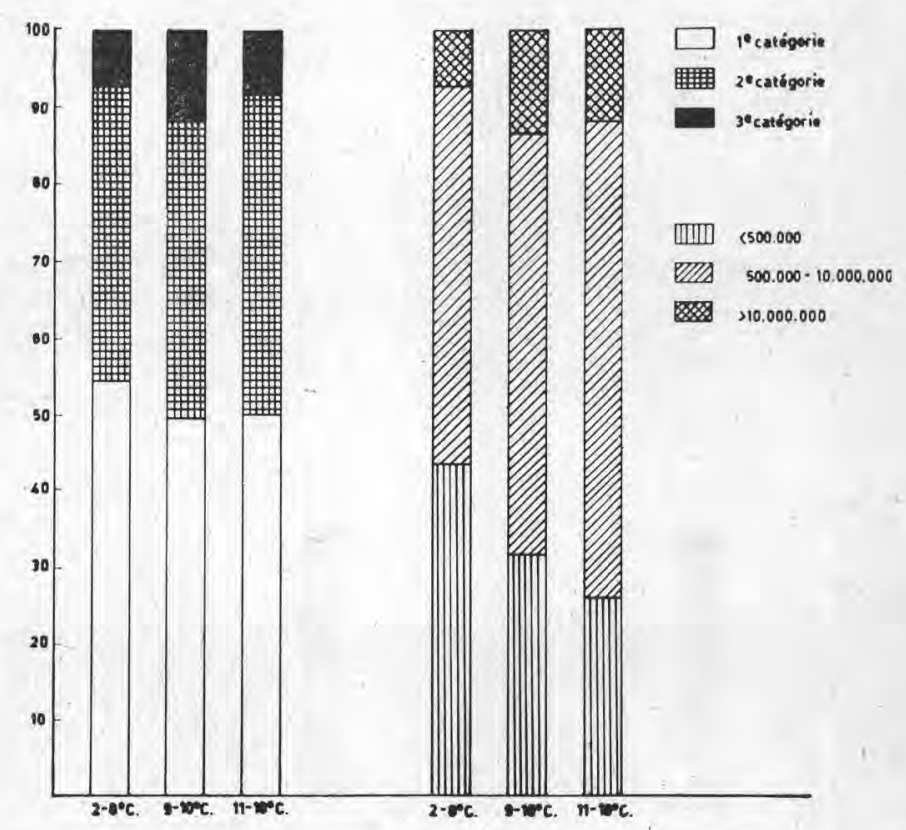

Fig. 3. - Répartition des échantillons en trois catégories, et en trois groupes selon le Clump Count pour trois zones de températures

Si on classe, pour les mêmes zones de températures, les laits eu trois groupes selon les temps de décoloration $(\leqslant 1 \mathrm{~h}, 1-5 \mathrm{~h}$, $>5 \mathrm{~h}$ ), on constate que le pourcentage des laits avec un temps de décoloration supérieur à $5 \mathrm{~h}$ varie avec la température : 47,3 p. 100

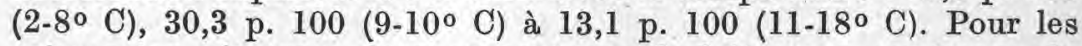
mêmes températures, les pourcentages de laits avec un temps de décoloration compris entre 1 et $5 \mathrm{~h}$, sont respectivement de 46,3 , 56,8 et 68,3 p. 100 . Quant aux laits médioeres avec un temps de décoloration égal ou inférieur à $1 \mathrm{~h}$, leur proportion sera respectivement 6,4 à 12,9 et 18,6 p. 100 .

En classant les laits selon le clump count on observe le même phénomène : lorsque la température augmente, il y a diminution. de la proportion de bons laits et augmentation de celle de laits de 
qualité moyenne ou médiocre. Pour les trois zones de température envisagées, la proportion des échantillons avec clump count inférieur à 500000 varie en sens inverse de la température : 43,5 p. $100\left(2-8^{\circ} \mathrm{C}\right)$ à 31,6 p. $100\left(9-10^{\circ} \mathrm{C}\right)$ et 22,6 p. $100\left(11-18^{\circ} \mathrm{C}\right)$. Par contre, la proportion de laits avec clump count compris entre 500000 et 10000000 s'élève avec la température : 49,4 p. 100, 55,5 p. 100 et 66 p. 100 respectivement; il en est de même pour les laits médiocres avec clump count supérieur à 10000000 qui passent de 7,1 p. $100\left(2-8^{\circ} \mathrm{C}\right), 12,9$ p. $100\left(9-10^{\circ} \mathrm{C}\right)$ à 11,4 p. $100\left(11-18^{\circ} \mathrm{C}\right)$.

Le tableau II donne les valeurs statistiques de l'influence de la température sur les tests étudiés. Il en ressort que les coefficients de corrélation entre température et le clump count sont petits, mais très significatifs. Les déviations standard sont grandes et les lignes de régression ont la même inclinaison et sont proches l'une de l'autre. Les coefficients de corrélation entre la température et le temps de décoloration sont grands et très significatifs, ceci par l'effet de compensation suivant la température extérieure.

Ces données statistiques confirment les conclusions de Thomé et coll. [12] qui estiment que l'épreuve au bleu de méthylène permet seulement de distinguer les laits mal refroidis ou récoltés malproprement, des autres laits qui peuvent être, soit de bonne, soit de mauvaise qualité bactériologique. Quant à Kandler [5], il considère aussi que l'épreuve au bleu de méthylène ne permet pas, lorsqu'ils ont été refroidis convenablement, de distinguer les laits de bonne qualité bactériologique de ceux de mauvaise qualité. Busse [2], quant à lui, conclut que si l'on prend l'épreuve au bleu de méthylène comme base de classification de lait, cela revient à ne tenir compte que de la teneur en bactéries lactiques car les bactéries sporulées et les coliformes n'influencent qu'accessoirement le résultat de cette épreuve. Simonart et coll. [8] ont confirmé que les laits à teneur élevée en leucocytes, contenant éventuellement de nombreux St. agalactiae, donnent en moyenne un résultat plus favorable à l'épreuve au bleu de méthylène que les laits normaux. Les calculs statistiques ultérieurs ont montré que les différences étaient significatives. D'après Baumgartner [1], non seulement la flore bactérienne du lait, mais aussi le nombre de leucocytes peuvent influencer favorablement ou défavorablement le temps de réduction, selon qu'il s'agit de lait de mélange ou de lait individuel. Singh et Marshall [9] ont constaté une diminution du nombre de germes dans le lait, avec un nombre croissant de leucocytes. Cette phagocytose se manifesterait déjà avec 10000 leucocytes par ml de lait. Cette diminution de germes pourrait expliquer un temps de réduction plus 1 favorable avec un nombre élevé de leucocytes.

Chalmers [3], à la suite d'autres auteurs, fait une critique de la méthode au bleu de méthylène; il considère que l'épreuve au bleu de méthylène ne donne guère d'indications sur les fautes 


\section{TABLEAU II}

INFLUENCE DE LA TEMPÉRATURE EXTÉRIEURE SUR LE TEMPS DE RÉDUCTION DU BLEU DE MÉTHYLìNE ET SUR LE CLUMP COUNT

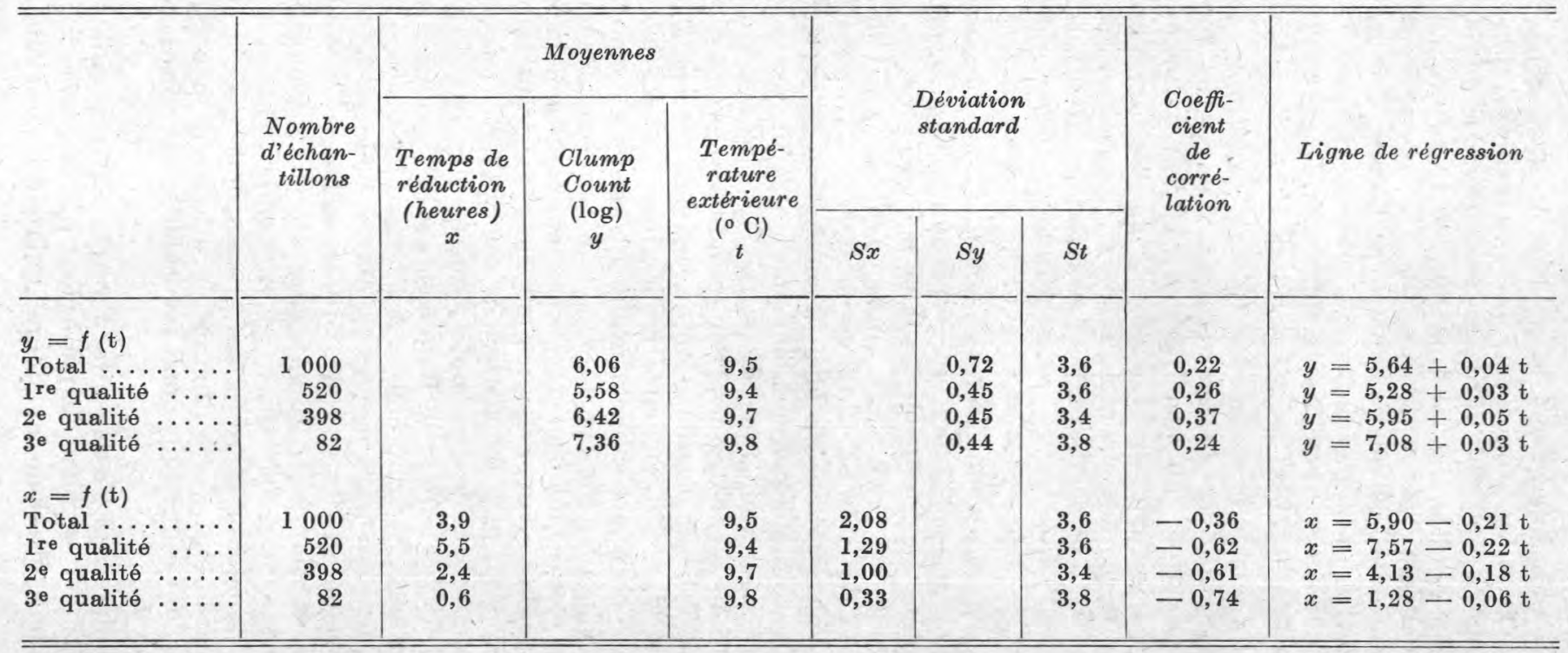


commises à la production du lait et qu'elle est sans utilité pour juger les laits individuels. Récemment, Swartling [11] souligne aussi les lacunes de cette épreuve.

Au regard des reproches qui sont faits à l'épreuve au bleu de méthylène en tant que base d'appréciation de la qualité bactériologique des laits, il s'indique de rechercher un autre moyen pour juger plus convenablement de cette qualité. Le clump count pourrait être appliqué dans ce but. La méthode est expéditive et. peu coûteuse; elle simplifie l'acheminement des échantillons au laboratoire car ceux-ci peuvent être additionnés d'un antiseptique. Elle permet d'effectuer une contre-analyse, alors que cela est exclu dans l'épreuve au bleu de méthylène ; en outre, la préparation microscopique constitue une preuve permanente du résultat. Une seule personne peut examiner sans fatigue un nombre très élevé d'échantillons, surtout lorsqu'il ne s'agit pas de faire un comptage précis, mais bien de répartir les échantillons en deux ou trois catégories suivant un seuil donné. Enfin, l'image microscopique peut donner des indications précieuses quant à la nature de la flore bactérienne.

\section{Résumé et conclusions}

Tout en tenant compte de la température extérieure, la qualité bactériologique de 1000 échantillons de lait de petit mélange a été contrôlée comparativement par l'épreuve au bleu de méthylène et par le dénombrement microscopique (clump count). Les résultats ont été confrontés avec ceux que donne le système de classification des laits d'après la réglementation belge.

L'analyse statistique des diverses données étudiées montre une grande dispersion des résultats. Elle met en évidence, entre autres, l'influence très significative de la température extérieure sur la qualité bactériologique. Dès lors un système de classification, qui est fonction de la température extérieure, ne permet pas d'apprécier la qualité intrinsèque du lait. Si, en outre, ce système se base sur l'épreuve au bleu de méthylène qui est, à juste titre, l'objet de critiques sévères, il est doublement aléatoire. Par contre, l'examen microscopique du lait, avec dénombrement des bactéries, donne des indications utiles tant hygiéniquement que techniquement, quant à la qualité bactériologique du lait.

\section{Summary}

While taking the outside temperature into account, the bacteriological quality of thousand herd milk samples has been controled comparatively by the methylene blue test and by the Direct 
Microscopic Clump Count. The results have been compared with those given by the milk classification system in accordance with the Belgian regulations.

Statistical analysis of the different data shows a great dispersion of the results and it makes obvious, among others, the significant influence of the outside tem perature upon the bacteriological quality. Consequently a classification system dependent on the outside temperature does not allow to appreciate the intrinsic quality of the milk. If, besides, this system is based on the methylene blue test, which is rightly subject to severe criticisms, it is twice aleatory. The microscopic examination of the milk, with the bacterial count, on the contrary, gives indications concerning the bacteriological quality of the milk, which are hygienically and technically useful.

\section{RÉFÉRENCES}

[1] Baumgartiner (H.). Schweiz. Milchztg, 1961, 87, 501.

[2] Busse (M.). Z. Lebensmitt. Untersuch. 1960, 113, 464-472.

[3] Chalmers (C. H.). Bacteria in relation to the milk supply, 4th ed., London, 1962.

[4] Dавван (R.) and Olson (J. C. jr.), J. Dairy Sc., 1965, 48, 770.

[5] Kandler (O.). Dtsch. Molkereiztg. 1959, 80, 1959-1962.

[6] Niusson (R.) und von Bockelmann (I.). XVII Int. Dairy Congr. München, 1966, A : 349-357.

[7] Overby (A. J.), Royal Vet. Agr. Coll. Copenhagen, Yearbook 1964, 1-33.

[8] Srmonart (P.), Poffé (R.) et Weckx (M.). XVIIe Int. Dairy Congr. München, 1966, A : 369-372.

[9] Singh (B.) and MaRShaLI (R. T.). J. Dairy Sc., 1965, 48, 769.

[10] Standard Methods for the Examination of Dairy Products 11th. Ed. N. Y., 1960.

[11] Swartiling (P.). Neth. Milk Dairy J., 1967, 21, 87-102.

[12] Тномé (K. E.), Samuerson (E. G.) und Mattsson (N.). Milchwissenschaft, 1955, 10, 232-238. 